\title{
MIMO Receiver Using Reduced Complexity Sequence Estimation With Channel Estimation and Tracking
}

\author{
Yau Hee Kho, Member, IEEE, and Desmond P. Taylor, Life Fellow, IEEE
}

\begin{abstract}
A sequence-based multiple-input-multiple-output receiver incorporating channel estimation and tracking for a frequency selective fading environment is developed. It employs near-maximum likelihood sequence estimation using the partitioned Viterbi algorithm and offers linearly increasing complexity with the number of transmit antennas. The receiver implements channel estimation and tracking using a vector-polynomial-based generalized recursive least squares algorithm. The resulting integrated receiver can operate in a continuously time-varying Rayleigh or Rician fading environment. Simulation results show that it offers a good tradeoff between complexity and performance. Further complexity reduction can be achieved using tentative decisions as reference signals to the estimator and a reduced complexity form of the estimator.
\end{abstract}

Index Terms-Channel estimation, equalizers, fading channels, mobile communication, multiple-input-multiple-output (MIMO) systems.

\section{INTRODUCTION}

I NTERSYMBOL interference (ISI), as encountered by wideband wireless systems, is a major cause of performance degradation in frequency-selective fading environments [1], and equalization is needed to improve system performance. Practical methods with low complexity such as minimum mean square error (MMSE) decision feedback equalization (DFE) can be used. However, performance tends to be poor, particularly in channels with deep spectral nulls [1]. Maximumlikelihood sequence estimation (MLSE) using the Viterbi algorithm (VA) is the optimum equalization method [2], [3].

Manuscript received August 28, 2007; revised March 6, 2008 and April 28, 2008. First published June 3, 2008; current version published February 17, 2009. The work of Y. H. Kho was supported in part by the University of Canterbury under a doctoral scholarship and by the Institution of Engineering and Technology (formerly the Institution of Electrical Engineers) under the Hudswell International Research Scholarship. The review of this paper was coordinated by Prof. X.-G. Xia.

Y. H. Kho is with NZi3: Wireless Research Centre, University of Canterbury, Christchurch 8140, New Zealand. He was previously with the Department of Electrical and Computer Engineering, University of Canterbury, Christchurch 8140, New Zealand (e-mail: yhkho@ieee.org).

D. P. Taylor is with the Department of Electrical and Computer Engineering, University of Canterbury, Christchurch 8140, New Zealand (e-mail: taylor@elec.canterbury.ac.nz).

Color versions of one or more of the figures in this paper are available online at http://ieeexplore.iee.org.

Digital Object Identifier 10.1109/TVT.2008.926589
However, its complexity exponentially increases with the length of the channel delay spread. In a multiple-input-multipleoutput (MIMO) system, MLSE requires the use of the vector VA (VVA) [4], which adds significant complexity to the receiver.

The complexity of MLSE using the VVA depends on the number of states in the ISI-induced trellis. In a MIMO context, this depends on the modulation constellation size $M$, the number of transmit antennas $T$, and the channel delay spread $L$ according to $M^{T L}$. For a given value of $M$, the MLSE complexity exponentially increases with both $T$ and $L$. For example, a $4 \times 4$ MIMO system $(T=4)$ that transmits quaternary phaseshift keying (QPSK signals; $M=4$ ), in a fading environment, assuming a channel delay spread of 2 symbols $(L=2)$ requires 65536 states for MLSE. For larger constellations and more transmit antennas, the number of states quickly grows out of hand. Moreover, in some channels, the delay spread is significantly longer, which further increases the complexity. Channel shortening filters that reduce the length of the effective channel impulse response (CIR) [5], reduced-state sequence estimation [6], and delayed decision feedback sequence estimation [7] can be used to reduce the number of states. However, there is still an exponential dependence on both $T$ and $L$.

Thus, reduced-complexity sequence estimation techniques that offer a linear increase in complexity with $T$ and/or $L$ are highly desired. In [8], a decision feedback MLSE scheme is proposed, where complexity linearly increases with $L$ according to $L M^{T}$. For the aforementioned example, the number of states would, then, be 512. Alternatively, a partitioned VA (PVA) with a linear increase in complexity with $T$ according to $T M^{L}$ is proposed in [9]. The number of states is, then, only 64 for the aforementioned example. The PVA results in linearly increasing complexity with $T$, which is more important in MIMO systems, so it is considered in this paper.

In [9], channel fading is assumed to be quasistatic, where the CIR is assumed to remain constant throughout the transmission of a signal frame but randomly varies from frame to frame. Training symbols are used at the beginning of each frame to estimate the CIR by using the least squares (LS) technique. The estimates are then used to equalize the remainder of the frame. Channel tracking is not used in [9], and the system performance tends to degrade in a continuously timevarying fading environment, particularly for longer frames. Here, we extend the PVA-based receiver in [9] to cope with continuous fading by incorporating channel estimation and 


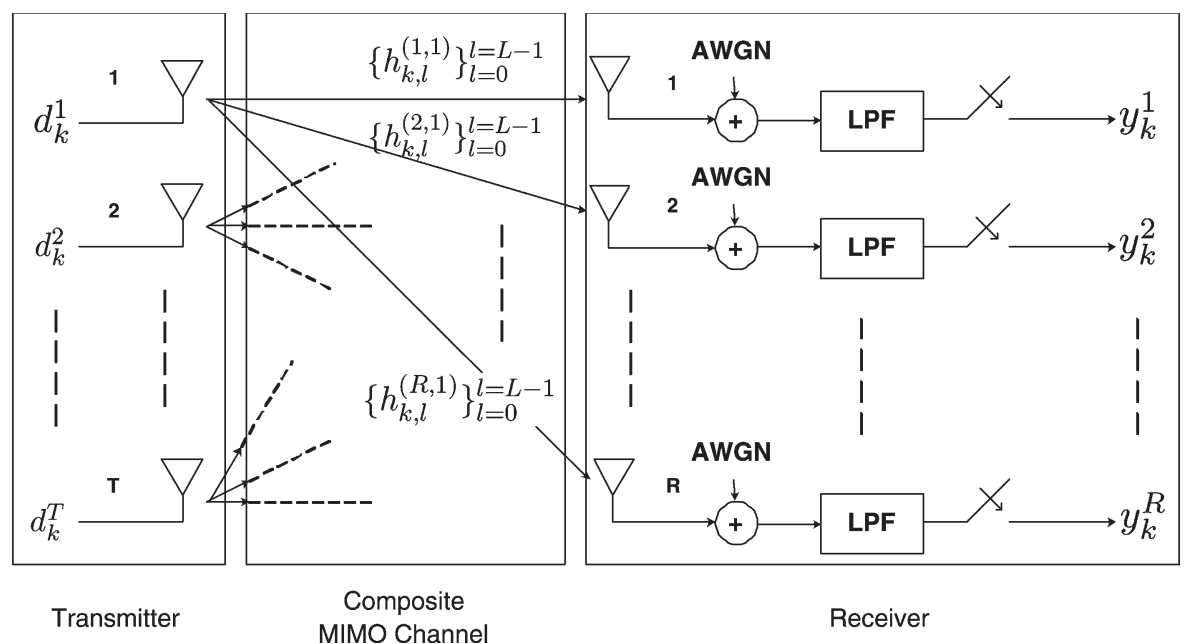

Fig. 1. General block diagram of a discrete-time $(T, R)$ MIMO communication system at time $k$.

tracking using a vector-polynomial-based generalized recursive least squares (VGRLS) channel estimator [10]. The resulting receiver is an implementable approximation to MLSE in MIMO channels and is among the first sequence-estimationbased receivers that explicitly incorporate dynamic channel estimation.

We describe the overall signal model in Section II. In Section III, we describe the proposed integrated receiver. Simulation results and discussions are presented in Section IV, followed by the conclusions in Section V.

\section{Signal Model}

We assume a MIMO system that transmits independent signals from each of $T$ antennas to $R \geq T$ receive antennas using a Vertical-Bell Laboratories-Layered-Space-Time (VBLAST)type transmission format [11]. Fig. 1 shows a discrete-time model for the system. At the receiver, each of the $R$ antennas observes a linear combination of the transmitted signals. The $j$ th symbol rate sample of the complex baseband received signal at time $k$ may be written as

$$
y_{k}^{(j)}=\sum_{i=1}^{T} \sum_{l=0}^{L-1} d_{k-l}^{(i)} h_{k, l}^{(j, i)}+n_{k}^{(j)}, \quad j=1,2, \ldots, R
$$

where $d_{k}^{(i)}$ is the $k$ th transmitted complex baseband $M$-ary data symbol from the $i$ th antenna, $\left\{h_{k, l}^{(j, i)}\right\}_{l=0}^{l=L-1}$ is the sampled fading dispersive composite $\mathrm{CIR}^{1}$ between the $i$ th transmit and $j$ th receive antennas at time $k$ with a delay spread of $L$ symbol periods, and $n_{k}^{(j)}$ is the sampled additive white Gaussian noise (AWGN) with variance $\sigma_{n}^{2}$.

With an oversampling factor of $N_{r} \geq 1$ so that sampling occurs every $T_{\mathrm{sym}} / N_{r}$ s, with $T_{\mathrm{sym}}$ being the symbol

\footnotetext{
${ }^{1}$ It is assumed to be the convolution of the transmit pulse shape and physical channel response.
}

period, we define vectors of $N_{r}$ samples in the $k$ th symbol period as

$$
\begin{aligned}
& \mathbf{y}_{k}^{(j)}=\left(\begin{array}{c}
y_{k, 0}^{(j)} \\
y_{k, 1}^{(j)} \\
\vdots \\
y_{k, N_{r}-1}^{(j)}
\end{array}\right), \quad \mathfrak{H}_{k, l}^{(j, i)}=\left(\begin{array}{c}
h_{k, l, 0}^{(j, i)} \\
h_{k, l, 1}^{(j, i)} \\
\vdots \\
h_{k, l, N_{r}-1}^{(j, j)}
\end{array}\right) \\
& \mathbf{n}_{k}^{(j)}=\left(\begin{array}{c}
n_{k, 0}^{(j)} \\
n_{k, 1}^{(j)} \\
\vdots \\
n_{k, N_{r}-1}^{(j)}
\end{array}\right) .
\end{aligned}
$$

Based on (1), we may write an oversampled (vector) form of the signal in the $k$ th symbol interval as

$$
\mathbf{y}_{k}^{(j)}=\sum_{i=1}^{T} \sum_{l=0}^{L-1} d_{k-l}^{(i)} \mathfrak{H}_{k, l}^{(j, i)}+\mathbf{n}_{k}^{(j)}, \quad j=1,2, \ldots, R .
$$

The MIMO received signal in (3) may, then, be expressed in matrix-vector form [9] as

$$
\mathbf{y}_{k}=\sum_{l=0}^{L-1} \mathbf{H}_{k, l} \mathfrak{d}_{k-l}+\mathbf{n}_{k}
$$

where

$$
\mathbf{y}_{k}=\left(\begin{array}{c}
\mathbf{y}_{k}^{(1)} \\
\mathbf{y}_{k}^{(2)} \\
\vdots \\
\mathbf{y}_{k}^{(R)}
\end{array}\right), \quad \mathfrak{d}_{k}=\left(\begin{array}{c}
d_{k}^{(1)} \\
d_{k}^{(2)} \\
\vdots \\
d_{k}^{(T)}
\end{array}\right), \quad \mathbf{n}_{k}=\left(\begin{array}{c}
\mathbf{n}_{k}^{(1)} \\
\mathbf{n}_{k}^{(2)} \\
\vdots \\
\mathbf{n}_{k}^{(R)}
\end{array}\right)
$$

and we define the $R N_{r} \times T$ channel matrix taps as

$$
\mathbf{H}_{k, l}=\left(\begin{array}{ccc}
\mathfrak{H}_{k, l}^{(1,1)} & \ldots & \mathfrak{H}_{k, l}^{(1, T)} \\
\vdots & \ddots & \vdots \\
\mathfrak{H}_{k, l}^{(R, 1)} & \ldots & \mathfrak{H}_{k, l}^{(R, T)}
\end{array}\right), \quad l=0,1,2, \ldots, L-1 .
$$




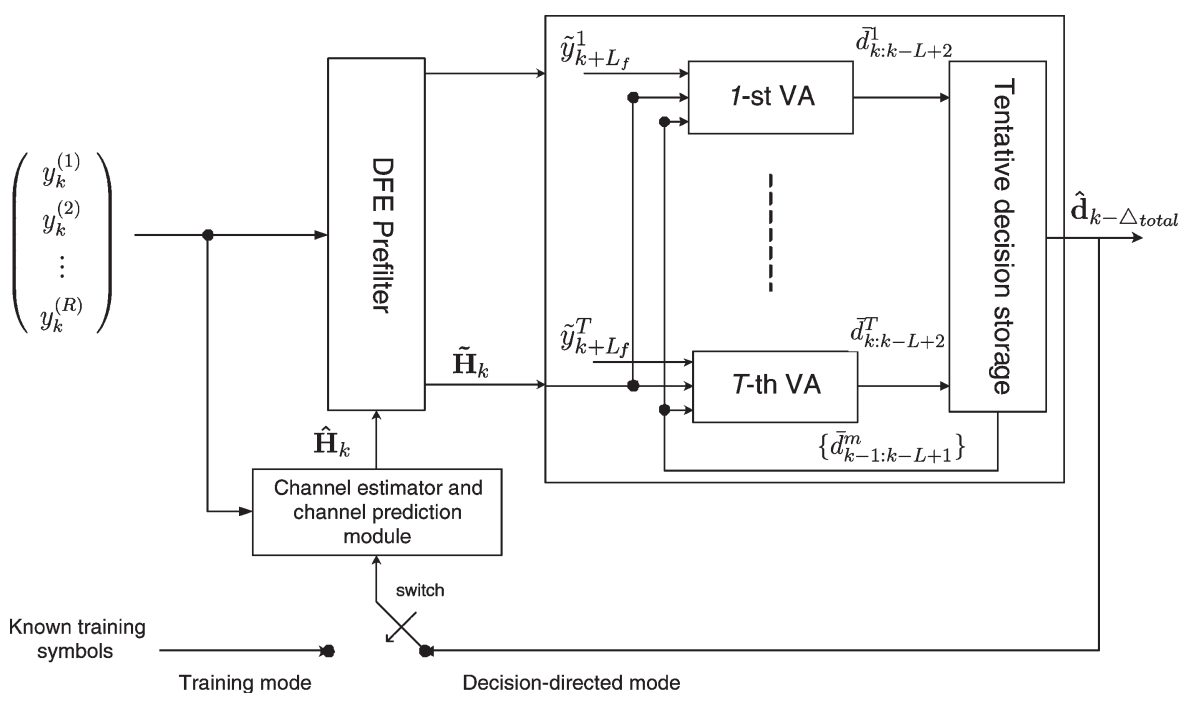

Fig. 2. Integrated sequence-based receiver that uses the PVA algorithm with VGRLS channel estimation and tracking for a continuously frequency-selective fading environment.

To facilitate the description of the VGRLS estimator [10], we reformulate (4). We observe that there are $L$ channel matrix taps. Following [10], we represent each matrix tap as a column vector by using the operator $\operatorname{vec}\left(\mathbf{H}_{k, l}\right)$ and stack the columns of $\mathbf{H}_{k}=\left[\mathbf{H}_{k, 0}, \ldots, \mathbf{H}_{k, L-1}\right]$ into a length $R N_{r} T L$ channel vector, i.e.,

$$
\begin{aligned}
\mathfrak{h}_{k}=\operatorname{vec}\left(\mathbf{H}_{k}\right) & \\
= & h_{k, 0,0}^{(1,1)} \cdots h_{k, 0, N_{r}-1}^{(1,1)} \cdots h_{k, 0,0}^{(R, 1)} \cdots h_{k, 0, N_{r}-1}^{(R, 1)}, \cdots \\
& h_{k, 0,0}^{(1, T)} \cdots h_{k, 0, N_{r}-1}^{(1, T)} \cdots h_{k, 0,0}^{(R, T)} \cdots h_{k, 0, N_{r}-1}^{(R, T)}, \cdots \\
& h_{k, L-1,0}^{(1,1)} \cdots h_{k, L-1, N_{r}-1}^{(1,1)} \cdots h_{k, L-1,0}^{(R, 1)} \\
& \quad \cdots h_{k, L-1, N_{r}-1}^{(R, 1)}, \ldots, h_{k, L-1,0}^{(1, T)} \cdots h_{k, L-1, N_{r}-1}^{(1, T)} \\
& \left.\cdots h_{k, L-1,0}^{(R, T)} \cdots h_{k, L-1, N_{r}-1}^{(R, T)}\right]^{t}
\end{aligned}
$$

where $t$ denotes matrix transposition. To ensure dimensional compatibility, we also define an $R N_{r} \times R N_{r} T L$ transmitted data matrix $\mathfrak{D}_{k}$ as

$$
\mathfrak{D}_{k}=\left[d_{k}^{(1)} \cdots d_{k}^{(T)}, \ldots, d_{k-L+1}^{(1)} \cdots d_{k-L+1}^{(T)}\right] \odot \mathbf{I}_{R N_{r}}
$$

where $\mathbf{I}_{R N_{r}}$ is the $R N_{r} \times R N_{r}$ identity matrix, and $\odot$ is the Kronecker product. We may, then, write (4) in the following compact form:

$$
\mathbf{y}_{k}=\mathfrak{D}_{k} \mathfrak{h}_{k}+\mathbf{n}_{k} .
$$

\section{PRoposed RECEIVER}

In this section, we develop the integrated sequence-based receiver by using the PVA algorithm with VGRLS channel estimation and tracking, as shown in Fig. 2. To do this, we replace the nontracking LS channel estimator in [9] with the
VGRLS estimator in [10]. The VGRLS estimator tracks the channel variation so that the channel estimates are time variant from symbol to symbol. There are two aspects that require attention, compared with the original structure proposed in [9]: 1) the symbol-by-symbol updating of the CIR estimates and its effect on the subsequent PVA operation and 2) the effect of the VA and DFE prefilter decision delays on the updating of the CIR estimates. The second aspect causes the VGRLS estimator to produce delayed channel estimates. We consider these in more detail in the following sections.

\section{A. Sequence Estimation Based on the PVA}

A major component of the PVA is a length $L_{f}$ prefilter that is used to provide linear estimates of each of the $T$ transmitted signal streams. Ideally, it should be a vector whitened matched filter (WMF) [2]. However, in practice, the vector WMF does not always exist [12]. Thus, the feedforward filter of a vector MMSE DFE is used [9]. We note that the DFE prefilter always exists, even when the WMF does not. It has been shown to approach WMF performance as the SNR and number of filter taps increase [12]. The prefilter compensates for precursor ISI and decouples the received signal vector into $T$ signal streams. Parallel VAs are then used to process these outputs to obtain equalized data estimates. Tentative decisions are made in each interval, and these are exchanged among the parallel VAs. For each transmitted signal stream, feedback terms that were estimated using the tentative decisions obtained in the previous interval from the other processors are used to cancel cross-interference effects of the other signal streams.

1) DFE Prefilter: In this section, we describe the derivation of the DFE prefilter. Following [9] we assume that a length $L_{f}$ fractionally spaced FIR feedforward filter with matrix taps is used as the prefilter, where $L_{f}$ is the support of the prefilter impulse response in symbols. Each tap is denoted by a $T \times R N_{r}$ matrix $\mathbf{F}_{k, m}$ for $m=0,1, \ldots, L_{f}-1$. The prefilter matrix 
taps can be expressed in a vector as $\mathbf{F}_{k}=\left[\mathbf{F}_{k, 0}, \ldots, \mathbf{F}_{k, L_{f}-1}\right]$. Prefiltering the received signal vector $\mathbf{y}_{k}$ in (4) gives

$$
\tilde{\mathbf{y}}_{k}=\sum_{m=0}^{L_{f}-1} \mathbf{F}_{k, m} \mathbf{y}_{k-m}=\sum_{n=0}^{L_{f}+L-2} \tilde{\mathbf{H}}_{k, n} \mathfrak{d}_{k-n}+\tilde{\mathbf{n}}_{k}
$$

where the prefiltered channel response and noise are, respectively, defined as

$$
\begin{aligned}
\tilde{\mathbf{H}}_{k, n} & =\sum_{m=0}^{L_{f}-1} \mathbf{F}_{k, m} \mathbf{H}_{k-m, n-m} \\
\tilde{\mathbf{n}}_{k} & =\sum_{m=0}^{L_{f}-1} \mathbf{F}_{k, m} \mathbf{n}_{k-m} .
\end{aligned}
$$

Over a block of $L_{f}$ symbol periods (corresponding to the length of the DFE prefilter), the received signal vectors can be written in the compact form as

$$
\mathbf{y}_{k+L_{f}-1: k}=\mathbf{C}_{k} \mathfrak{d}_{k+L_{f}-1: k-L+1}+\mathbf{n}_{k+L_{f}-1: k}
$$

where $\mathbf{C}_{k}$ is the convolution matrix in (14), shown at the bottom of the page.

To facilitate the derivation of the DFE prefilter and the structure of the PVA trellis, we initially assume that the channel responses, i.e., the elements in (14), are available. In reality, these are obtained through channel estimation, which we will deal with later.

We partition (14) into three sections of $T\left(L_{f}-1\right), T$, and $T(L-1)$ columns that correspond to $\mathbf{H}_{k \text {,fut }}, \mathbf{H}_{k \text {,pres }}$ and $\mathbf{H}_{k \text {,past }}$. The first $T\left(L_{f}-1\right)$ columns represent the $L_{f} R N_{r} \times$ $T\left(L_{f}-1\right)$ matrix $\mathbf{H}_{k \text {,fut }}$, which represents the filter response from "future" symbols. The $T$ columns represent the $L_{f} R N_{r} \times$ $T$ matrix $\mathbf{H}_{k, \text { pres }}$, which represent the current transmitted symbols, and the last $T(L-1)$ columns represent the $L_{f} R N_{r} \times$ $T(L-1)$ matrix $\mathbf{H}_{k \text {,past }}$, which represent the previously transmitted symbols. Then, (13) can be written as

$$
\begin{aligned}
\mathbf{y}_{k+L_{f}-1: k}= & \left(\begin{array}{ll}
\mathbf{H}_{k, \text { fut }} \quad \mathbf{H}_{k, \text { pres }} \quad \mathbf{H}_{k, \text { past }}
\end{array}\right) \\
& \times \mathfrak{d}_{k+L_{f}-1: k-L+1}+\mathbf{n}_{k+L_{f}-1: k} \\
= & \mathbf{H}_{k, \text { fut }} \mathfrak{d}_{k+L_{f}-1: k+1}+\mathbf{H}_{k, \text { pres }} \mathfrak{d}_{k} \\
& +\mathbf{H}_{k, \text { past }} \mathfrak{d}_{k-1: k-L+1}+\mathbf{n}_{k+L_{f}-1: k} .
\end{aligned}
$$

In deriving the prefilter, we employ the methodology for designing an MMSE DFE, where the feedforward and feedback filter coefficients are jointly derived and optimized. We assume that correct past decisions are available (i.e., $\hat{\mathfrak{d}}_{k-1: k-L+1}=$ $\left.\mathfrak{d}_{k-1: k-L+1}\right)$ so that (15) can be used to write a vector DFE estimate as

$$
\begin{aligned}
\hat{\mathfrak{d}}_{k}= & \mathbf{F}_{k} \mathbf{y}_{k+L_{f}-1: k}-\mathbf{F}_{k} \mathbf{H}_{k, \text { past }} \mathfrak{d}_{k-1: k-L+1} \\
= & \mathbf{F}_{k} \mathbf{H}_{k, \text { fut }} \mathfrak{d}_{k+L_{f}-1: k+1}+\mathbf{F}_{k} \mathbf{H}_{k, \text { pres }} \mathfrak{d}_{k} \\
& +\mathbf{F}_{k} \mathbf{n}_{k+L_{f}-1: k} \\
= & {\left[\tilde{\mathbf{H}}_{k, 0}, \tilde{\mathbf{H}}_{k, 1}, \ldots, \tilde{\mathbf{H}}_{k, L_{f}-2}\right] \mathfrak{d}_{k+L_{f}-1: k+1} } \\
& +\tilde{\mathbf{H}}_{k, L_{f}-1} \mathfrak{d}_{k}+\tilde{\mathbf{n}}_{k+L_{f}-1: k}
\end{aligned}
$$

where $\tilde{\mathbf{H}}_{k}=\left[\tilde{\mathbf{H}}_{k, 0}, \tilde{\mathbf{H}}_{k, 1}, \ldots, \tilde{\mathbf{H}}_{k, L_{f}-1}\right]$ is the time-variant prefiltered CIR that was defined by (11). The prefiltered noise $\tilde{\mathbf{n}}_{k+L_{f}-1: k}$ is assumed to be Gaussian and white [9], which is a requirement for the PVA's use of the VA. We note that, in some cases (e.g., in an overloaded system when $T>R$ ), the noise may not be white. In such a case, the colored-noise version of the VA [13] can be used.

The FIR vector DFE uses the forward filter $\mathbf{F}_{k}$ that minimizes the mean square error (MSE) $E\left\{\left\|\hat{\mathfrak{d}}_{k}-\mathfrak{d}_{k}\right\|^{2}\right\}$. It is shown that the MSE is minimized when this filter satisfies [9]

$$
\left(\left[\mathbf{H}_{k, \text { fut }}, \mathbf{H}_{k, \text { pres }}\right]\left[\mathbf{H}_{k, \text { fut }}, \mathbf{H}_{k, \text { pres }}\right]^{H}+\sigma_{n}^{2} \mathbf{I}\right) \mathbf{F}_{k}^{H}=\mathbf{H}_{k, \text { pres }} \text {. }
$$

Since $\left(\left[\mathbf{H}_{k, \text { fut }}, \mathbf{H}_{k, \text { pres }}\right]\left[\mathbf{H}_{k, \text { fut }}, \mathbf{H}_{k, \text { pres }}\right]^{H}+\sigma_{n}^{2} \mathbf{I}\right)$ is Hermitian and positive definite, (17) can be solved efficiently using the Cholesky decomposition.

The advantage of using the DFE prefilter is that the prefiltered channel matrix taps $\tilde{\mathbf{H}}_{k, n}$ for $n=0,1, \ldots, L_{f}-1$ are $T \times T$ matrices that are independent of the number of receive antennas $R$ and oversampling factor $N_{r}$ [9]. This means that increasing $R$ or $N_{r}$ will increase the complexity of solving (17) but not the complexity of the VA. By examining (16), it is shown that the MSE is minimized when the first $L_{f}-1$ prefiltered channel taps $\left[\tilde{\mathbf{H}}_{k, 0}, \tilde{\mathbf{H}}_{k, 1}, \ldots, \tilde{\mathbf{H}}_{k, L_{f}-2}\right]$ approximate zero matrices, and the tap $\tilde{\mathbf{H}}_{k, L_{f}-1}$ approximates the identity matrix. With these approximations, the $\tau$ th output of the prefilter for $\tau=1,2, \ldots, T$ can be written as

$$
\begin{aligned}
\tilde{y}_{k+L_{f}-1}^{(\tau)}= & \sum_{m=1}^{T} \sum_{n=0}^{L_{f}+L-2} \tilde{h}_{k, n}^{(\tau, m)} d_{k+L_{f}-1-n}^{(m)}+\tilde{n}_{k+L_{f}-1}^{(\tau)} \\
\approx & \sum_{m=1}^{T} \sum_{n=0}^{L-1} \tilde{h}_{k, n+L_{f}-1}^{(\tau, m)} d_{k-n}^{(m)}+\tilde{n}_{k+L_{f}-1}^{(\tau)} \\
\approx & \sum_{n=0}^{L-1} \tilde{h}_{k, n+L_{f}-1}^{(\tau, \tau)} d_{k-n}^{(\tau)} \\
& +\sum_{m \neq \tau} \sum_{n=1}^{L-1} \tilde{h}_{k, n+L_{f}-1}^{(\tau, m)} d_{k-n}^{(m)}+\tilde{n}_{k+L_{f}-1}^{(\tau)}
\end{aligned}
$$

$$
\mathbf{C}_{k}=\left(\begin{array}{cccccc}
\mathbf{H}_{k+L_{f}-1,0} & \cdots & \mathbf{H}_{k+L_{f}-1, L-1} & 0 & \cdots & 0 \\
0 & \mathbf{H}_{k+L_{f}-2,0} & \cdots & \mathbf{H}_{k+L_{f}-2, L-1} & 0 & \cdots \\
\vdots & \ldots & 0 & & & \vdots \\
0 & \cdots & & \mathbf{H}_{k, 0} & \cdots & \mathbf{H}_{k, L-1}
\end{array}\right)
$$


This equation shows that the $\tau$ th prefilter output value is a function of the $L$ most recent symbols from the $\tau$ th transmitter $d_{k: k-L+1}^{(\tau)}$ and $(L-1)$ delayed symbols $\left\{d_{k-1: k-L+1}^{(m)}\right\}_{m \neq \tau}$ from each of the other transmitters. This approximation is used to generate inputs to the PVA algorithm, and the structure of (18) is exploited to develop the PVA algorithm.

2) Trellis Structure: As we have previously described, the inputs to the PVA algorithm are the prefiltered received signal $\left(\tilde{\mathbf{y}}_{k+L_{f}}\right)$ and prefiltered channel response $\left(\tilde{\mathbf{H}}_{k}\right)$. There are $T$ outputs from the prefilter, so $T$ VAs are employed to process them in parallel [9]. Consider an estimate of the $\tau$ th transmitted symbol sequence $\hat{d}_{k: k-L+1}^{(\tau)}$ for $\tau=1,2, \ldots, T$. Divide the estimate into two overlapping sections that define the $M^{L-1}$ "states" $\hat{d}_{k-1: k-L+1}^{(\tau)}$ and $\hat{d}_{k: k-L+2}^{(\tau)}$, where each state corresponds to a possible symbol combination. Let $\mathbf{i}$ indicate a particular previous state $\hat{d}_{k-1: k-L+1}^{(\tau)}$, and let $\mathbf{j}$ indicate a particular current state $\hat{d}_{k: k-L+2}^{(\tau)}$. The branch metric of the VA that is used for the $\tau$ th trellis at each time $k$, which corresponds to the state transition $(\mathbf{i}, \mathbf{j})$, is given by

$$
\lambda^{\tau}(\mathbf{i}, \mathbf{j}, k)=\left|\tilde{y}_{k+L_{f}-1}^{(\tau)}-\sum_{n=0}^{L-1} \tilde{h}_{k, n+L_{f}-1}^{(\tau, \tau)} \hat{d}_{k-n}^{(\tau)}-\phi(k, \tau)\right|^{2}
$$

where

$$
\phi(k, \tau)=\sum_{m \neq \tau} \sum_{n=1}^{L-1} \tilde{h}_{k, n+L_{f}-1}^{(\tau, m)} \bar{d}_{k-n}^{(m)}
$$

is the feedback term that was estimated using the tentative decisions $\left\{\bar{d}_{k-n}^{(m)}\right\}_{m \neq \tau}$ that the other VAs made during the previous symbol time. The summation in (19) is an estimate of the $\tau$ th transmitter's contribution to the observed value in $\tilde{y}_{k+L_{f}-1}^{(\tau)}$.

\section{B. VGRLS Channel Estimation and Tracking}

The VGRLS channel estimator in [10] is developed based on the theory of polynomial prediction by using Taylor series expansions on the underlying channel responses in the time domain (known as the $t$-power series in [14]). It can offer comparable performance with an optimal Kalman filter (KF)based [15] estimator without requiring a priori any secondorder channel and noise statistics. As these statistics often require a long measurement time to acquire [16], they add a layer of complexity to the estimation process. Furthermore, a $\mathrm{KF}$ estimator cannot operate in a Rician fading environment without requiring reconfiguration of its state transition matrix [17] to accommodate the nonrandom mean components. This is not required for a VGRLS estimator, because it employs fixed polynomial coefficients that are known a priori. Due to its Kalman-like behavior, the VGRLS estimator can track the temporal variation of the CIR reasonably well. Therefore, it offers a nice tradeoff between complexity and performance compared to an optimal KF estimator [10]. We summarize the VGRLS estimator here.
TABLE I

POLYNOMIAL COEFFICIENTS OF VARIOUS ORDER AND LENGTH

\begin{tabular}{|c|c|c|}
\hline Length P & Order $N$ & Polynomial Coefficients $\left\{a_{1}, a_{2}, \cdots, a_{P}\right\}$ \\
\hline \hline 2 & 0 & $\{1 / 2,1 / 2\}$ \\
2 & 1 & $\{2,-1\}$ \\
3 & 0 & $\{1 / 3,1 / 3,1 / 3\}$ \\
3 & 1 & $\{4 / 3,1 / 3,-2 / 3\}$ \\
3 & 2 & $\{3,-3,1\}$ \\
\hline
\end{tabular}

In [10], a state-space model of the channel state vector with unforced dynamics based on polynomial predictors of length $P$ and order $N$ was developed as

$$
\mathbf{h}_{k+1}=\mathbf{U h}_{k}
$$

where

$$
\mathbf{h}_{k}=\left[\mathfrak{h}_{k}^{t}, \mathfrak{h}_{k-1}^{t}, \ldots, \mathfrak{h}_{k-P+1}^{t}\right]^{t}
$$

is the $\left(R N_{r} T L P \times 1\right)$ channel state vector at time $k$, and the $\left(R N_{r} T L P \times R N_{r} T L P\right)$ state transition matrix is given by

$$
\mathbf{U}=\left(\begin{array}{ccccc}
\mathfrak{U}_{1} & \mathfrak{U}_{2} & \cdots & \mathfrak{U}_{P-1} & \mathfrak{U}_{P} \\
& \mathbf{I}_{R N_{r} T L(P-1)} & \cdots & & \mathbf{0}_{A, B}
\end{array}\right) .
$$

Here, $\mathbf{I}_{m}$ is the $(m \times m)$ identity matrix, and $\mathbf{0}_{A, B}$ is the $(A \times B)$ null matrix, where $A=R N_{r} T L(P-1)$, and $B=$ $R N_{r} T L$. Each $\left(R N_{r} T L \times R N_{r} T L\right)$ polynomial predictor matrix is given by $\mathfrak{U}_{r}=a_{r} \mathbf{I}_{R N_{r} T L}$, where $a_{r}$ is the $r$ th coefficient of a polynomial predictor with length $P$ and $\operatorname{order} N$, as shown in Table I. These polynomial coefficients can be derived a priori without requiring channel statistics. Note that, when $P=1$ and $N=0$, the VGRLS estimator reduces to a conventional vector RLS estimator.

Similar to the KF algorithm, the VGRLS algorithm consists of two main operations: 1) time update and 2) prediction. By defining an augmented data vector

$$
\mathbf{d}_{k}=\left[\begin{array}{lll}
\mathfrak{D}_{k} & \mid & \mathbf{0}_{R N_{r}, R N_{r} T L(P-1)}
\end{array}\right]
$$

with $\mathfrak{D}_{k}$ being given by (8), the update equations for the VGRLS algorithm [10] may be written as

$$
\begin{aligned}
\mathbf{K}_{k} & =\mathbf{P}_{k / k-1} \mathbf{d}_{k}^{H}\left(\mathbf{I}_{R N_{r}}+\mathbf{d}_{k} \mathbf{P}_{k / k-1} \mathbf{d}_{k}^{H}\right)^{-1} \\
\mathbf{P}_{k / k} & =\left(\mathbf{I}_{R N_{r} T L P}-\mathbf{K}_{k} \mathbf{d}_{k}\right) \mathbf{P}_{k / k-1} \\
\hat{\mathbf{h}}_{k / k} & =\hat{\mathbf{h}}_{k / k-1}+\mathbf{K}_{k}\left(\mathbf{y}_{k}-\mathbf{d}_{k} \hat{\mathbf{h}}_{k / k-1}\right) .
\end{aligned}
$$

The one-step prediction is written as

$$
\begin{aligned}
\hat{\mathbf{h}}_{k+1 / k} & =\mathbf{U} \hat{\mathbf{h}}_{k / k} \\
\mathbf{P}_{k+1 / k} & =\lambda^{-1} \mathbf{U} \mathbf{P}_{k / k} \mathbf{U}^{H}
\end{aligned}
$$

where $\hat{\mathbf{h}}_{k / k-1}$ is the estimate of the channel state vector at time $k$ based on $(k-1)$ prior received samples, $\lambda$ is the RLS 
"forget factor," $\mathbf{K}_{k}$ is analogous to the Kalman gain vector [15], and $\mathbf{P}_{k / k}$ is the so-called "intermediate" matrix. ${ }^{2}$ Note that the performance of the VGRLS estimator has been evaluated and compared with a KF-based estimator in [10]. Its performance is found to be slightly worse but comparable to that of the KFbased estimator.

In decision-directed mode, the VGRLS estimator in the receiver employs the detected symbols ${ }^{3} \hat{\mathbf{d}}_{k}$ from the PVA output in place of $\mathbf{d}_{k}$, which is the known training symbol vector. Due to the decision delay $\Delta$ of the VAs, these are delayed by $\Delta$ symbol periods. Furthermore, as the received signal $\mathbf{y}_{k}$ passes through the length $L_{f}$ DFE prefilter, a decision delay of $L_{f}-1$ is introduced. Therefore, there is a total delay of $\Delta_{\text {total }}=L_{f}+\Delta-1$ symbols with respect to the input of the receiver (see Fig. 2).

Using these delayed symbols, together with the received vector $\mathbf{y}_{k-\Delta_{\text {total }}}$ and $P$ previously estimated channel vectors, the VGRLS estimator produces a delayed channel estimate ${ }^{4}$ $\hat{\mathbf{h}}_{k-\Delta_{\text {total }}+1}$. However, to calculate the length $L_{f}$ prefilter taps of the PVA at time $k+1$, up-to-date estimated channel vectors that correspond to the most recent $L_{f}$ symbols should be used. The VGRLS estimator provides one of these $L_{f}$ estimates, and the subsequent $L_{f}-1$ estimates are still required. One simple method that we can employ is to assume that the channel remains constant over these $L_{f}$ symbol periods so that $\hat{\mathbf{h}}_{k-\Delta}=\cdots=\hat{\mathbf{h}}_{k-\Delta_{\text {total }}+2}=\hat{\mathbf{h}}_{k-\Delta_{\text {total }}+1}$, where $\hat{\mathbf{h}}_{k-\Delta_{\text {total }}+1}$ is available from the VGRLS estimator. However, strictly speaking, this may apply only to a very slowly fading channel.

One alternative approach that we can use is to predict the $L_{f}-1$ channel vectors. Here, we employ a vector polynomial channel prediction module that is similar to the module in [10] to predict the estimated channel vectors. The underlying structure of the VGRLS estimator uses a $t$-power series expansion [14] to model the channel fading process as an $N$ th-order polynomial series, so the polynomial-based state transition matrix $\mathbf{U}$ in (23) is readily available. It is then straightforward to compute the predicted channel estimates as

$$
\begin{array}{ccc}
\hat{\mathbf{h}}_{k-\triangle_{\text {total }}+2} & = & \mathbf{U} \hat{\mathbf{h}}_{k-\triangle_{\text {total }}+1} \\
\vdots & = & \vdots \\
\hat{\mathbf{h}}_{k-\triangle} & = & \mathbf{U} \hat{\mathbf{h}}_{k-\triangle-1} .
\end{array}
$$

We note that the complexity of the VGRLS estimator can be reduced significantly by replacing the online Riccatti computation of (26) with an offline precomputed matrix. This reduces the complexity from $\mathcal{O}\left(\left(R N_{r} T L P\right)^{3}\right)$ real operations per iteration in the highest order terms to $\mathcal{O}\left(\left(R N_{r} T L P\right)^{2}\right)$ and results in a reduced complexity algorithm known as the vectorgeneralized least mean squares (VGLMS) estimator [18]. Thus,

\footnotetext{
${ }^{2} \mathbf{P}_{k / k}$ is the inverse input autocorrelation matrix in a conventional RLS algorithm.

${ }^{3}$ Here, the detected symbol vector $\hat{\mathfrak{d}}_{k}$ is rearranged into $\hat{\mathbf{d}}_{k}$, which is a matrix with an appropriate dimension as required by the VGRLS estimator, as in (24).

${ }^{4}$ For brevity, we simplify the notation $\hat{\mathbf{h}}_{k-\Delta_{\text {total }}+1 / k-\Delta_{\text {total }}}$ to $\hat{\mathbf{h}}_{k-\Delta_{\text {total }}+1 \text {. }}$
}

we have also simulated an integrated receiver that employs the VGLMS estimator and PVA algorithm. We note that it can be used in place of the VGRLS estimator but with some loss in receiver performance.

Upon obtaining new channel estimates at time $k$, we formulate $\hat{\mathbf{C}}_{k}$ the estimated convolution matrix of (14) and use it to solve for the prefilter coefficients in (17). We process a block of $L_{f}$ received signal vectors to obtain (18) and use the channel estimates to obtain the prefiltered channel taps $\tilde{\mathbf{H}}_{k, n}$ for $n=0,1, \ldots, L_{f}-1$. Then, we compute the branch metrics for each of the $T$ parallel VAs as in (19) and (20). After a VA decision delay of $\Delta$, an estimate of the transmitted vector $\hat{\mathbf{d}}_{k-\Delta_{\text {total }}}$ is produced $^{5}$ as the PVA output.

\section{RECEIVER OPERATION}

The receiver is operated in two modes: 1) a training mode and 2) a decision-directed data transmission mode. Each transmitted frame consists of $L_{t}$ training symbols, followed by $L_{d}$ data symbols. The receiver operation is described as follows.

\section{A. Training Mode}

Here, only the VGRLS estimator is operated using a training sequence of length $L_{t}$ according to the following.

Step 1) Initiate the VGRLS algorithm with an all-zero estimated channel vector $\hat{\mathbf{h}}_{1 / 0}$ and "intermediate" matrix $\mathbf{P}_{1 / 0}=\delta^{-1} \mathbf{I}_{R N_{r} T L P}$, where $\delta$ is a small positive real constant, and $\mathbf{I}_{R N_{r} T L P}$ is an identity matrix with dimension $R N_{r} T L P \times R N_{r} T L P$. Using the observation vector $\mathbf{y}_{k}$, compute the Kalman gain in (25), update the "intermediate" matrix in (26), and update the estimated channel vector in (27).

Step 2) Compute the one-step predicted channel vector and the one-step predicted "intermediate" matrix in (29).

Step 3) With each observation vector $\mathbf{y}_{k}, k \geq 2$, repeat Steps 2) and 3) until the end of the training sequence.

Following training, the receiver switches to a decisiondirected operation.

\section{B. Decision-Directed Mode}

In this mode, the DFE prefilter and PVA algorithm are operated in tandem with the VGRLS estimator and the channel prediction module during the $L_{d}$ data transmission period. In this mode, the feedback terms are calculated using tentative decisions from the VAs. Assuming $\hat{\mathbf{d}}_{k-\Delta_{\text {total }}}$ to be the data vector at time $k$, the receiver operation may be described as follows.

Step 1) With $\hat{\mathbf{h}}_{k-\Delta_{\text {total }}}$ being available at time $k$, operate the VGRLS estimator to produce $\hat{\mathbf{h}}_{k-\Delta_{\text {total }}+1}$ at time $k+1$ by using the PVA output vectors

\footnotetext{
${ }^{5}$ Note that $\Delta_{\text {total }}=\Delta+L_{f}-1$
} 


\author{
$\hat{\mathbf{d}}_{k-\Delta_{\text {total }}}, \ldots, \hat{\mathbf{d}}_{k-\Delta_{\text {total }}-L+1}$, received vector \\ $\mathbf{y}_{k-\Delta_{\text {total }}}$, and $P$ previously estimated channel \\ vectors.
}

Step 2) Predict the next $L_{f}-1$ channel vectors as in (30). Recall that each estimated vector $\hat{\mathbf{h}}$ follows the structure of (22) with $P$ vector elements $\hat{\mathfrak{h}}$, where each element or subvector has $R N_{r} T L$ components, as shown in (7).

Step 3) Following (2) and (6), the estimated convolution matrix $\hat{\mathbf{C}}_{k+1}$ at time $k+1$ can be obtained and structured.

Step 4) Calculate the prefilter coefficients $\mathbf{F}_{k+1}$ of the vector DFE in (17) and the prefiltered CIR estimate as $\tilde{\mathbf{H}}_{k+1}=\hat{\mathbf{C}}_{k+1} \otimes \mathbf{F}_{k+1}$, where $\otimes$ is the convolution operator.

Step 5) Prefilter the received signals to obtain the $T$ outputs in (18).

Step 6) Calculate the feedback terms in (20) by using tentative decisions from the previous symbol period.

Step 7) For each of the $T$ parallel VAs, calculate the branch metric in (19) and advance the algorithms by one time step.

Step 8) Output the data decisions $\hat{\mathbf{d}}_{k-\Delta_{\text {total }}+1}$.

Step 9) At each succeeding time instant, repeat Steps 1)-8) until the end of the frame.

\section{Simulation Results and Discussions}

We now evaluate the performance of the integrated PVAbased receiver. We assume an uncoded VBLAST-type [11] MIMO system, where each transmitter uses the same $M$-ary modulation, pulse shape, carrier frequency, and transmit power. This condition is considered to be one of the more difficult detection scenarios, because only channel differences can be used to separate the spatially multiplexed cochannel signals [9]. We assume independent wide-sense stationary uncorrelated scattering subchannels with similar fading conditions on each. The fading processes are assumed to follow Clarke's fading model [19] and are simulated according to [20]. Each subchannel is assumed to have a uniform power delay profile with $L=3$ rays.

Independent QPSK signal streams are transmitted from each of $T$ antennas. A raised cosine filter with a roll-off of 0.99 is used at the transmitter, with its response being truncated to $\pm 2 T$. An ideal low-pass filter with sufficient bandwidth for accommodating the Doppler faded signal is employed at the receiver inputs, and an oversampling factor of $N_{r}=2$ is used. We mimic GSM specifications where each frame at each antenna consists of $L_{t}=26$ training symbols and $L_{d}=$ 116 data symbols, unless stated otherwise. We also include $L_{f}+L-2=7$ termination symbols to ensure a known trellis end state. Both the training symbols and the data symbols are randomly generated. We use a prefilter, with $L_{f}=6$ taps, and assume a VA decision delay of $5 L=15$ symbol periods. For each SNR point, the simulation is carried out until 200 errors are accumulated overall.

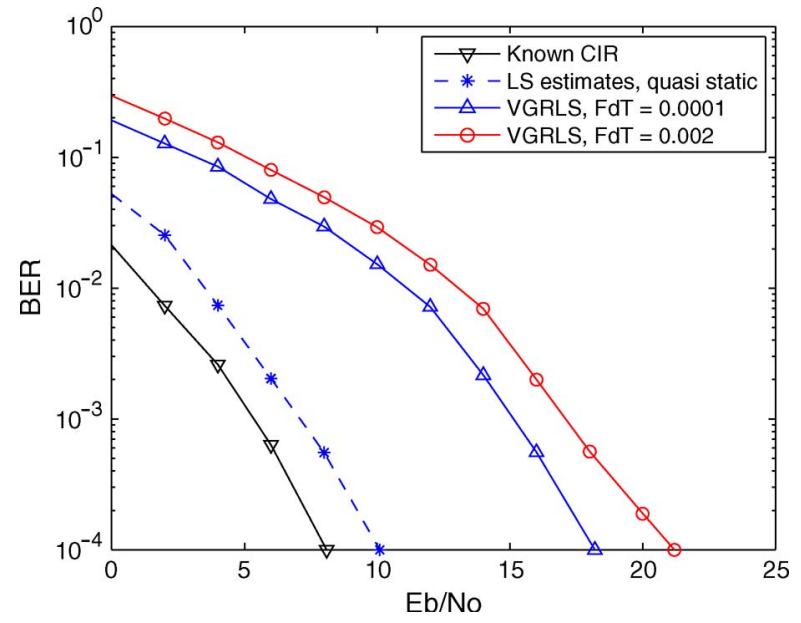

Fig. 3. BER of the PVA receiver when operating with the VGRLS estimator for a $(2,2)$ MIMO system with a normalized Rayleigh fading rate of 0.0001 and 0.002 . The performance of the PVA with known CIR and when using LS channel estimates in a quasistatic-fading channel is also plotted for reference.

The SNR is defined as the received $E_{b} / \sigma_{n}^{2}$ per receive antenna. The average received energy per bit $E_{b}$ is defined as

$$
E_{b}=\frac{\sigma_{d}^{2} \cdot \sigma_{h}^{2} \cdot T}{\log _{2} M}
$$

where $\sigma_{d}^{2}=1$ is the transmitted symbol power, and $\sigma_{h}^{2}=1$ is the normalized variance of the composite channel responses. For a given SNR, the complex AWGN variance of $\sigma_{n}^{2}$ can be calculated as

$$
\sigma_{n}^{2}=\frac{E_{b}}{10^{\frac{S N R}{10}}} .
$$

We compare the performance of the integrated PVA receiver with a VVA [4] receiver that also operates with a VGRLS estimator to provide the CIR for MLSE. A VGRLS estimator with a predictor length of $P=3$ and polynomial order of $N=2$ is used. Both receivers are operated under the same fading conditions. For simplicity, we restrict the simulations to a $(2,2)$ system. For this scenario, the VVA receiver requires 4096 states, whereas the PVA receiver requires a total of 128 states, i.e., a 32 -fold reduction in complexity.

Fig. 3 illustrates the effect of VGRLS channel estimation error upon the average bit error rate (BER) performance of the integrated PVA receiver in a $(2,2)$ MIMO fading channel with normalized fade rates $f_{d} T$ of 0.002 and 0.0001 , where $f_{d}$ is the maximum Doppler frequency. The performance of the PVA receiver with known CIR and by using LS channel estimation in a quasistatic fading channel [9] is also included for reference. For $f_{d} T=0.002$, we note that the VGRLS channel estimation error degrades the performance by about $12 \mathrm{~dB}$ at a BER of $10^{-3}$ compared with the known CIR case. At a BER of $10^{-4}$, the performance is slightly worse as the curve is shown to start flooring in a very gradual manner. This degradation is largely due to the dynamics of the fading as it introduces errors in 


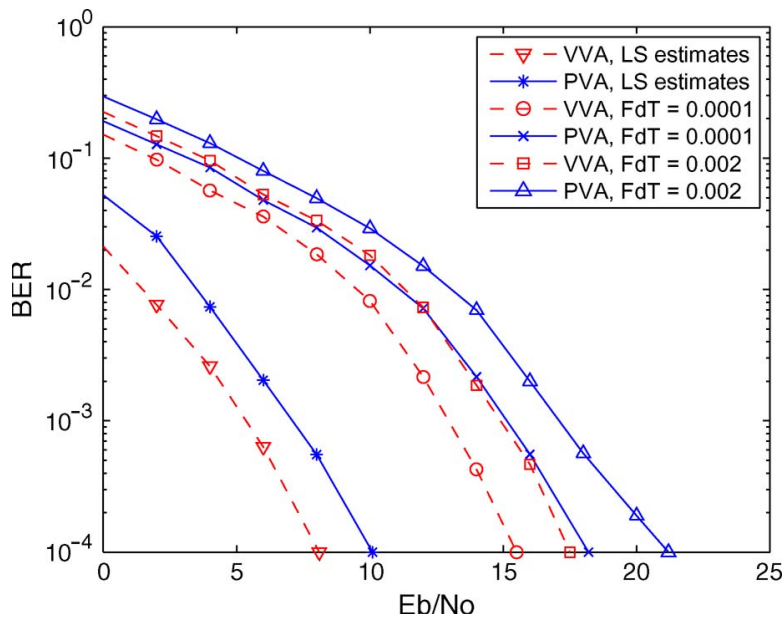

Fig. 4. BER of the PVA and VVA receivers when operating with the VGRLS estimator for a $(2,2)$ MIMO system with normalized Rayleigh fading rates of 0.0001 and 0.002 . The performance of PVA and VVA when using LS estimates in a quasistatic-fading channel is also plotted for comparison.

the channel estimates, which, in turn, affects the accuracy of the DFE prefilter calculation, the prefiltered received signal and estimated CIR, and the tentative decisions. This is evident in slower fading with $f_{d} T=0.0001$, where the error floor disappears, and the degradation is reduced to only $10 \mathrm{~dB}$ at both the aforementioned BER values. Compared with the quasistatic fading case, a continuously time-varying fading channel has a significant effect on the performance of the integrated PVA receiver.

Fig. 4 shows the BER performance of the integrated PVA receiver and a VVA receiver, which are both for a $(2,2)$ MIMO system, each operating with a VGRLS estimator in similar Rayleigh fading channels with $f_{d} T$ values of 0.002 and 0.0001 . The performance of a $(2,2)$ system that uses LS channel estimates in a quasistatic-fading channel [9] is also simulated for reference. We observe that the difference between the PVA and VVA receivers at $f_{d} T=0.002$ is about $4 \mathrm{~dB}$ at a BER of $10^{-4}$. This difference is only $2 \mathrm{~dB}$ [9] at the same BER value in a quasistatic-fading channel. The additional degradation is due to the time-varying channel estimation error (on prefilter calculation and the subsequent effects), because at $f_{d} T=0.0001$, the error floor disappears, and the difference is reduced to less than $3 \mathrm{~dB}$. The results show that the integrated PVA receiver can offer near-MLSE detection in a continuous-fading environment, which it achieves at a significantly lower complexity.

The decision delay $\Delta$ of the VA is typically set at $5 L$ symbol periods [1]. For our simulations with $L=3$, the VA decision delay is, therefore, 15 symbol periods. We investigate two options for overcoming this long latency: 1) using a shorter VA decision delay, which we propose to be $2 L=6$ symbol periods, and 2) employing the tentative decisions, which correspond to a zero VA decision delay, as reference signals for the VGRLS estimator in each symbol period. Note that in both cases, there is still a DFE prefilter decision delay of $L_{f}-1=5$ symbol periods, where channel prediction is required.

The BER performance of the two options at $f_{d} T=0.0001$ is shown in Fig. 5. We note that performance, when using

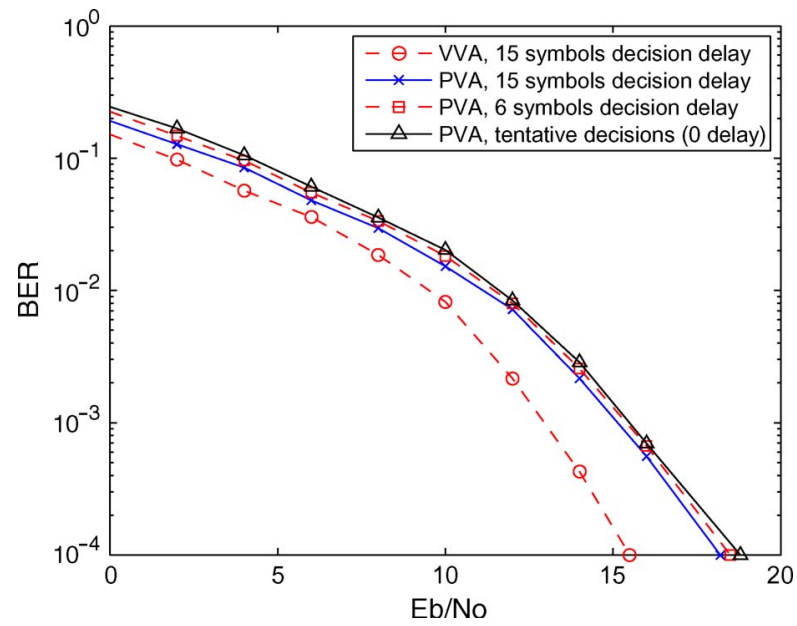

Fig. 5. BER of the PVA and VVA receivers when operating with the VGRLS estimator for a $(2,2)$ MIMO system with a normalized Rayleigh fading rate of 0.0001 . The decision delays of the VAs are given as indicated. Using tentative decisions (i.e., zero delay) has negligible degradation in performance.

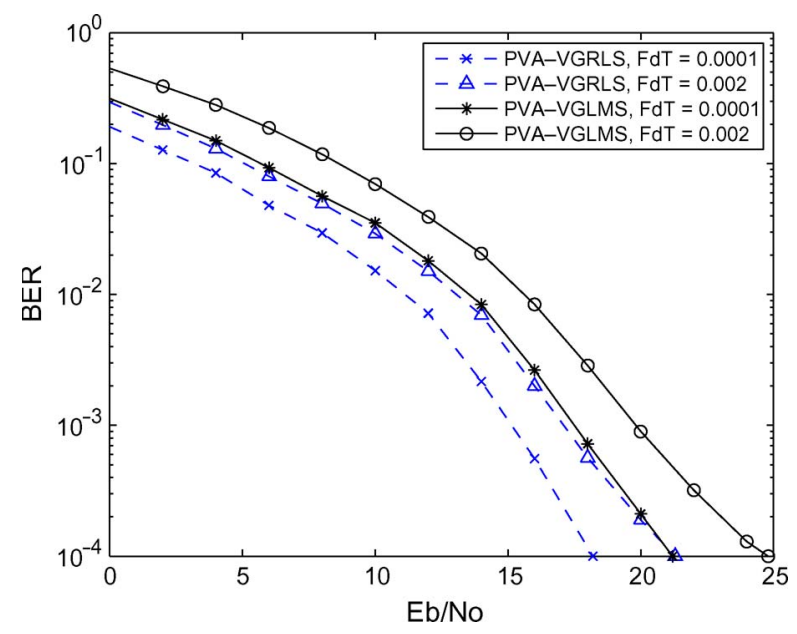

Fig. 6. BER of a PVA-based receiver that uses VGRLS and VGLMS estimators for a $(2,2)$ MIMO system with normalized Rayleigh fade rates of 0.0001 and 0.002 .

a decision delay of 6 symbols, is slightly better than when using the zero-delay tentative decisions, although the difference is only about $0.2 \mathrm{~dB}$. Both performances are, as expected, very slightly worse than when using the original delay of 15 symbols. However, this very small degradation shows that zero-delay tentative decisions can be used, and there is little justification for using the longer VA decision delay.

The complexity of channel estimation and tracking can be further reduced by using the VGLMS estimator, at least, in slow fading. To see this case, we evaluate the performance of an integrated PVA receiver that uses the VGLMS estimator. The receiver is similarly operated for a $(2,2)$ Rayleigh fading with normalized fade rates of 0.0001 and 0.002 . As shown in Fig. 6, the VGLMS-based receiver performs 2-3 dB worse than the VGRLS-based receiver. This degradation in performance should be expected because of the simplification in the estimation process. We also note that, for the VGLMS-based receiver at a fade rate of 0.002 , the error rate performance is shown to gradually start flooring more obviously than in 


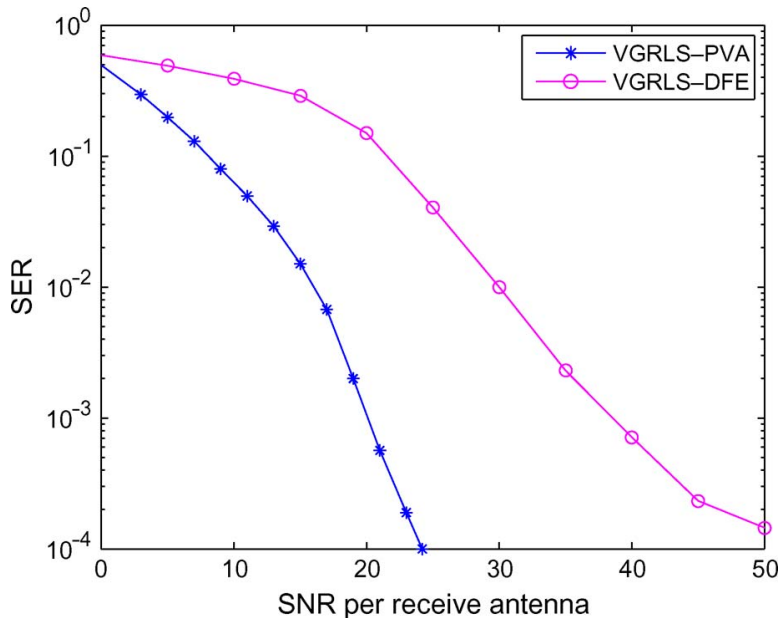

Fig. 7. Performance comparison of the integrated PVA receiver with a DFEbased receiver [10] that operates in a MIMO system with a normalized Rayleigh fading rate of 0.002 .

the case of the VGRLS-based receiver. However, within the range of SNR and conditions studied, the VGLMS-based receiver offers an attractive tradeoff between performance and complexity.

The PVA algorithm, being a sequence-based detection method that uses the VA, is more complicated than symbolbased detection methods such as the vector DFE. As such, the performance of the integrated PVA receiver is expected to be better than that of a DFE-based integrated receiver that also employs the VGRLS estimator under the same channel condition. Fig. 7 shows a comparison of the average symbol error rate (SER) performance of the integrated PVA receiver with the integrated DFE receiver in [10] in a Rayleigh fading with a normalized fade rate $f_{D} T=0.002$. It clearly shows the superiority of the PVA receiver over the DFE receiver, where the performance is about $17 \mathrm{~dB}$ better at an SER of $10^{-3}$. The SER results in [10] have also shown that the performance of the DFE receiver that uses the VGRLS estimator is within $1-3 \mathrm{~dB}$ of that obtained using an optimum KF-based estimator. The performance difference that is attributed to using VGRLS and KF-based estimators for a PVA receiver is within the same margin.

\section{CONCLUSION}

We have developed a reduced-complexity, sequence-based receiver for a MIMO system that can operate in a continuously time-varying fading environment. The overall receiver, which is an implementable approximation to MLSE in MIMO channels, has been implemented by combining the PVA algorithm with the VGRLS channel estimator. It is among the first attempts that explicitly incorporate dynamic channel estimation in the context of sequence estimation receivers. Simulation results have shown that the integrated PVA-based receiver can offer near-MLSE performance compared with a VVA-based receiver, which also uses a VGRLS estimator, and at a significantly lower complexity in terms of the total number of trellis states. Simulations have also shown that using zero-delay tentative decisions results in negligible performance loss. The complexity in channel estimation can be further reduced by using a VGLMS estimator instead of the VGRLS approach, and the simulation results that we have provided for the range of SNR and conditions studied illustrate the tradeoff between performance and complexity.

We have noted that it is possible to extend the receiver structure to accommodate a larger MIMO system [e.g., a $(3,3)$ or $(4,4)$ MIMO system] where the tradeoff would be more significant. It is also possible to increase the system throughput by employing a higher order signaling format (e.g., 16-QAM). However, these are beyond the scope of this paper and will be investigated separately.

\section{REFERENCES}

[1] J. G. Proakis, Digital Communications, 4th ed. Boston, MA: McGrawHill, 2001.

[2] G. Forney, "Maximum-likelihood sequence estimation of digital sequences in the presence of intersymbol interference," IEEE Trans. Inf. Theory, vol. IT-18, no. 3, pp. 363-378, May 1972.

[3] G. Forney, "The Viterbi algorithm," Proc. IEEE, vol. 61, no. 3, pp. 268 278, Mar. 1973.

[4] W. van Etten, "Maximum likelihood receiver for multiple channel transmission systems," IEEE Trans. Commun., vol. COM-24, no. 2, pp. 276-283, Feb. 1976.

[5] N. Al-Dhahir, "FIR channel-shortening equalizers for MIMO ISI channels," IEEE Trans. Commun., vol. 49, no. 2, pp. 213-218, Feb. 2001.

[6] M. V. Eyuboglu and S. U. H. Qureshi, "Reduced-state sequence estimation with set partitioning and decision feedback," IEEE Trans. Commun., vol. 36, no. 1, pp. 13-20, Jan. 1988.

[7] A. Duel-Hallen and C. Heegard, "Delayed decision-feedback sequence estimation," IEEE Trans. Commun., vol. 37, no. 5, pp. 428-436, May 1989.

[8] M. N. Patwary, P. B. Rapajic, and J. Choi, "Decision feedback MLSE for spatially multiplexed MIMO frequency selective fading channel," Proc. Inst. Elect. Eng.-Commun., vol. 153, no. 1, pp. 39-48, Feb. 2006.

[9] C. L. Miller, D. P. Taylor, and P. T. Gough, "Estimation of co-channel signals with linear complexity," IEEE Trans. Commun., vol. 49, no. 11, pp. 1997-2005, Nov. 2001.

[10] Y. H. Kho and D. P. Taylor, "MIMO channel estimation and tracking based on polynomial prediction with application to equalization," IEEE Trans. Veh. Technol., vol. 57, no. 3, pp. 1585-1595, May 2008.

[11] G. J. Foschini, G. D. Golden, R. A. Valenzuela, and P. W. Wolniansky, "Simplified processing for high spectral efficiency wireless communication employing multi-element arrays," IEEE J. Sel. Areas Commun., vol. 17, no. 11, pp. 1841-1852, Nov. 1999.

[12] C. L. Miller and D. P. Taylor, "Digital prefiltering for multipleinput, multiple-output receivers," in Proc. ICC, Jun. 2001, vol. 6, pp. 1696-1699.

[13] G. Ungerboeck, "Adaptive maximum-likelihood receiver for carriermodulated data-transmission systems," IEEE Trans. Commun., vol. COM-22, no. 5, pp. 624-636, May 1974.

[14] P. A. Bello, "Characterization of randomly time-variant linear channels," IEEE Trans. Commun. Syst., vol. CS-11, no. 4, pp. 360-393, Dec. 1963.

[15] S. S. Haykin, Adaptive Filter Theory, 4th ed. Englewood Cliffs, NJ: Prentice-Hall, 2002.

[16] B. D. Hart and D. P. Taylor, "Maximum-likelihood synchronization, equalization, and sequence estimation for unknown time-varying frequency-selective Rician channels," IEEE Trans. Commun., vol. 46, no. 2, pp. 211-221, Feb. 1998.

[17] L. M. Davis, I. B. Collings, and R. J. Evans, "Coupled estimators for equalization of fast-fading mobile channels," IEEE Trans. Commun., vol. 46, no. 10, pp. $1262-1265$, Oct. 1998.

[18] Y. H. Kho and D. P. Taylor, "Reduced complexity MIMO channel estimation and equalization using apolynomial-predictor-based vector GLMS algorithm," in Proc. IEEE PIMRC Conf., Sep. 2007, pp. 1-5.

[19] W. C. Jakes, Microwave Mobile Communications, W. C. Jakes, Ed. New York: Wiley, 1974.

[20] D. Verdin and T. C. Tozer, "Generating a fading process for the simulation of land-mobile radio communications," Electron. Lett., vol. 29, no. 23, pp. 2011-2012, Nov. 1993. 


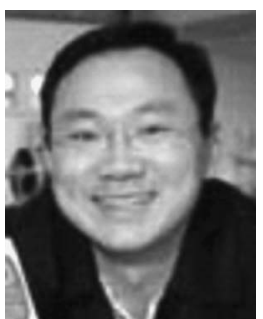

Yau Hee Kho (S'06-M'08) was born in Kuching, Sarawak, Malaysia, in 1974. He received the B.Eng. (Hons. 1) and Ph.D. degrees in electrical and computer engineering from the University of Canterbury, Christchurch, New Zealand, in 1997 and 2008, respectively.

From 1998 to 2002, he was an Electrical Design Engineer in Singapore. He is currently a Postdoctoral Researcher with the NZi3: Wireless Research Centre at the University of Canterbury. His research interests include estimation, equalization, and signal processing of digital wireless communications, in particular for MIMO systems.

Dr. Kho is a member of the Institution of Engineering and Technology (IET, formerly the IEE, U.K.). He served on the IET Council and Technical and Professional Board from 2006 to 2008. He was awarded first prize for the IET Write Around The World (WATW) competition in 2001 and third prize for the postgraduate section of the IEEE Region 10 Student Paper Contest in 2007.

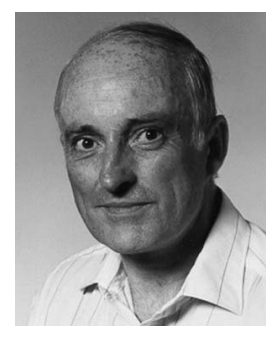

Desmond P. Taylor (M'65-SM'90-F'94-LF'07) was born in Noranda, QC, Canada, on July 5, 1941. He received the B.Sc.(Eng.) and M.Sc.(Eng.) degrees from Queen's University, Kingston, ON, Canada, in 1963 and 1967, respectively, and the Ph.D. degree in electrical engineering from McMaster University, Hamilton, ON, in 1972

From July 1972 until June 1992, he was with the Communications Research Laboratory and the Department of Electrical Engineering, McMaster University. In July 1992, he joined the University of Canterbury, Christchurch, New Zealand, where he is currently the Tait Professor of communications. His research interests include digital wireless communications systems (in particular, the development of robust bandwidthefficient modulation and coding techniques and the development of iterative algorithms for joint equalization and decoding of the fading dispersive channels in mobile radio communications), problems in synchronization, multiple access, and networking. He is the author or coauthor of approximately 220 published papers and is the holder of two U.S. patents in spread spectrum communications.

Dr. Taylor is a Fellow of the Royal Society of New Zealand, the Engineering Institute of Canada, and the Institute of Professional Engineers of New Zealand. $\mathrm{He}$ is the recipient of the 2001 S. O. Rice Award for the Best Transactions Paper in Communication Theory. 